\title{
Shock wave induced vaporization of porous solids
}

\author{
Andy H. Shen, a) Thomas J. Ahrens, and John D. O'Keefe \\ Lindhurst Laboratory of Experimental Geophysics, Seismological Laboratory, \\ California Institute of Technology, Pasadena, California 91125
}

(Received 9 May 2002; accepted 28 January 2003)

\begin{abstract}
Strong shock waves generated by hypervelocity impact can induce vaporization in solid materials. To pursue knowledge of the chemical species in the shock-induced vapors, one needs to design experiments that will drive the system to such thermodynamic states that sufficient vapor can be generated for investigation. It is common to use porous media to reach high entropy, vaporized states in impact experiments. We extended calculations by Ahrens [J. Appl. Phys. 43, 2443 (1972)] and Ahrens and O'Keefe [The Moon 4, 214 (1972)] to higher distentions (up to five) and improved their method with a different impedance match calculation scheme and augmented their model with recent thermodynamic and Hugoniot data of metals, minerals, and polymers. Although we reconfirmed the competing effects reported in the previous studies: (1) increase of entropy production and (2) decrease of impedance match, when impacting materials with increasing distentions, our calculations did not exhibit optimal entropy-generating distention. For different materials, very different impact velocities are needed to initiate vaporization. For aluminum at distention $(m)<2.2$, a minimum impact velocity of $2.7 \mathrm{~km} / \mathrm{s}$ is required using tungsten projectile. For ionic solids such as $\mathrm{NaCl}$ at distention $<2.2,2.5 \mathrm{~km} / \mathrm{s}$ is needed. For carbonate and sulfate minerals, the minimum impact velocities are much lower, ranging from less than 1 to $1.5 \mathrm{~km} / \mathrm{s}$. (C) 2003 American Institute of Physics. [DOI: 10.1063/1.1563035]
\end{abstract}

\section{INTRODUCTION}

Collision events and the resulting shock compressions are one of the most fundamental processes in planetary formation and evolution. They also play an important role in affecting the planetary climatic system on a planet such as Earth. ${ }^{1-3}$ During collision events, kinetic energy is converted into internal energy and such conversion changes the thermodynamic state of the colliding materials. Depending on the thermodynamic state reached and the release path taken, the system may end up in a solid, liquid, vapor, or plasma state. ${ }^{4}$ The amount of vapor released and chemical species in the released vapor, especially those materials containing water and hydroxyl, carbonate and sulfate groups, are of particular interests to planetary scientists as well as geologists. ${ }^{5,6}$ Previously, Boslough et al. ${ }^{7}$ and Tyburczy et $a l .{ }^{8}$ used a gun to launch a metal projectile into mineral specimens and the vapor products were collected and subsequently analyzed by gas source mass spectrometry. More recently, the vapor products generated from a hypervelocity impact of electrostatically accelerated microspheres upon metal targets were studied using time-of-flight mass spectrometry to simulate dust impact on a metal target. ${ }^{9-11}$ Such results were used to develop the dust detectors and analyzers on board of the Galileo, Cassini, and Stardust solar system probes. Recently, Yang and Ahrens ${ }^{12}$ have conducted velocity interferometer system for any reflector (VISAR) experiments on both calcite and anhydrite specimens and Gupta et $a l .{ }^{13}$ derived the incipient and complete vaporization pressures from these laser interferometric measurements. How-

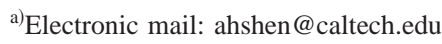

ever, in situ mass spectrometric experiment of the impact vaporization products has not been conducted using mineral samples.

On the other hand, previous simulation studies of the impact vaporization were focused on investigating impact cratering processes using hydrodynamic codes. ${ }^{14,15}$ Few studies focused on the thermodynamic states of materials after impact. Krieger at the RAND cooperation, Santa Monica, CA, conducted thermodynamic calculations for a series of materials including $\mathrm{CaCo}_{3},{ }^{16} \mathrm{CaO},{ }^{17} \mathrm{Mg}-\mathrm{Si}-\mathrm{O},{ }^{18}$ $\mathrm{SiC},{ }^{19} \mathrm{Al}-\mathrm{Si}-\mathrm{O}$ systems, ${ }^{20}$ and $\mathrm{Al} .{ }^{21}$ Recently, modern molecular dynamics studies have been performed on laser ablation processes, especially useful in analytical organic chemistry using matrix-assisted laser desorption ionization method. $^{22,23}$ Ahrens $^{24}$ and Ahrens and O'Keefe ${ }^{25}$ thermodynamically modeled porous materials for laboratory impact experiments. They calculated the entropies associated with the shocked states in metals, minerals, and common rocks. They observed that minerals and rocks are readily vaporizable at impact velocities less than $5 \mathrm{~km} / \mathrm{s}$ and they also noted the existence of an optimal distention in the entropy production for a specific material. In their model, they assumed the linear $U s-U p$ relation in porous materials to perform their impedance match calculations. Here, we explicitly require only a linear $U s-U p$ relation for nonporous solid materials and conduct thermodynamic modeling of the states of shocked porous materials upon projectile impact in the laboratory. We extended earlier calculations ${ }^{24,25}$ to distentions up to five and we improved the previous calculations by directly solving a modified Mie-Grüneisen equation of state for the porous material. Special interest is placed on minerals containing carbonates, sulfates, and hydroxyl groups that are of relevance to planetary climate 


\section{MODELING METHODS}

We assume that shocked states are thermodynamic equilibrium states and we limit our calculations to states with shock temperatures less than $10000 \mathrm{~K}$. Above such temperatures, significant ionization of gaseous elements takes place at ambient pressures and free electrons start to behave classically and begin to take up a significant fraction of the thermal energy. Hence, the simple treatment of Grüneisen parameter used in our modeling needs to be significantly modified, if peak shock temperature above $10000 \mathrm{~K}$ is reached. ${ }^{26} \mathrm{We}$ also ignored the effect of the presence of heterogeneous heating and hot spots in porous material under strong shock. These phenomena have been investigated ${ }^{27-29}$ and the main effect due to the presence of these nonequilibrium nonlinear phenomena is to create highly localized and concentrated heat and entropy sources within the shocked porous material. The presence of these highly localized hot spots will promote more vapor production and may cause additional chemical reactions in the vapor state. Such heat production can now be quantified to a certain degree and the shock temperature can be estimated ${ }^{29}$ but their effect on vapor production is less well established. Previous investigations (e.g., see Ref. 29 and references therein) concluded that nonequilibrium thermodynamics of energetic powders mainly affect the reaction initiation. The current study neglecting the nonequilibrium phenomena can be considered as a conservative estimate for vapor generation in a porous material.

In our calculations, the simulation grid included compression and distention. The compression is defined as:

$$
\eta=1-V_{H} / V_{0}=1-\rho_{0} / \rho_{H},
$$

where $V_{H}, \rho_{H}$ are the specific volume and density associated with a Hugoniot state and $V_{0}, \rho_{0}$ are the specific volume and density of initial nonporous crystalline material. The distention is defined as:

$$
m=V_{00} / V_{0}=\rho_{0} / \rho_{00},
$$

where $V_{00}$ and $\rho_{00}$ are the specific volume and density of the same material with different porosity. This study covered $m$ from 1 to 5 and $\eta$ from -0.5 to 0.5 .

The peak shock pressure associated with each grid point can then be calculated from a Mie-Grüneisen equation of state. One can then calculate the shock temperature using the existing thermodynamic data and the peak shock pressure. The entropy associated with each Hugoniot state can then be calculated from the shock pressure and temperature for each compression and distention grid point. Finally, with known compression and shock pressure, the shock velocity and particle velocity of each Hugoniot state can be calculated. Such calculation further allows us to solve an impedance match equation for each compression-distention grid point.

Many materials studied in our modeling undergo single or multiple phase transitions. In order to simplify the modeling, we adopted an iterative procedure ${ }^{30}$ to establish Hugoniots for the metastable high-pressure phase or high pressure phase assemblage. Therefore, the transformation energy and entropy are involved only in the shock temperature and entropy calculations.

\section{A. Hugoniot pressure and temperature calculations}

Assuming a Mie-Grüneisen equation of state, using the principal isentrope as a reference curve, the Hugoniot in the $P-\rho$ space for a crystalline material is given as the following: ${ }^{4}$

$$
P_{H}=\left(P_{S}(K-1)-2 \varepsilon_{S} \rho_{H}\right) /\left(K-\rho_{H} / \rho_{0}\right),
$$

in which, $P_{H}$ is the Hugoniot pressure, $P_{S}$ is the pressure along the principal isentrope at density $\rho_{H}$. Here, $K \equiv 1$ $+2 / \gamma$ where $\gamma$ is the Grüneisen parameter and $\varepsilon_{S}$ is the internal energy along the principal isentrope. The Grüneisen parameter is modeled with a volume dependence: ${ }^{31,32}$

$$
\gamma=\Gamma_{0} \exp \left(\frac{q_{0}}{q^{\prime}}\left(\frac{V_{H}}{V_{0}}\right)^{q^{\prime}}-1\right),
$$

where

$$
\frac{d \ln (\gamma)}{d \ln (V)}=q_{0}\left(\frac{V_{H}}{V_{0}}\right)^{q^{\prime}}=q \text { and } q^{\prime}=\frac{d \ln (q)}{d \ln (V)} .
$$

We used the theoretical value compatible with the linear $U s-U p$ relation for the zero-compression Grüneisen parameter, $\Gamma_{0}$, as the following: ${ }^{32}$

$$
\Gamma_{0}=\frac{162 s^{2}-360 s+215}{18 s},
$$

where $s$ is the slope from the linear $U_{s}-U_{p}$ relationship $\left(U_{s}=C_{0}+s \cdot U_{p}\right)$ of the crystalline material. ${ }^{33}$ The reasons for choosing the Eq. (4b) formulation versus other theoretical values are (1) for many high pressure phases, data for their Grüneisen parameter is unavailable and (2) such choice allows us to generate a self-consistant theoretical parameter set for many high-pressure phases. This parameter set includes Grüneisen parameters, the bulk modulus, and the pressure derivative of the bulk modulus. ${ }^{33}$

With minor modification, Eq. (3) can be extended to describe the Hugoniot for distended materials:

$$
P_{m}=\left(P_{S}(K-1)-2 \varepsilon_{S} \rho_{H}\right) /\left(K-\rho_{H} / \rho_{00}\right),
$$

where $P_{m}$ is the Hugoniot pressure for the porous material with initial density of $\rho_{00}$. The meaning of other symbols are exactly the same as in Eqs. (3) and (5), except the reference density is now changed to that of the porous material. This equation allows one to model the normal Hugoniot curves as well as the inverted Hugoniot curves at high porosity.

The pressure along the principal isentrope $\left[P_{s}\right.$ in Eqs. (3) and (5)] can be expressed in a third-order BirchMurnaghan equation of state and the internal energy change along the principal isentrope $\left[\varepsilon_{s}\right.$ in Eqs. (3) and (5) $]$ is $^{32}$

$$
\varepsilon_{s}=9 V_{0} K_{0 S}\left[f^{2} / 2+a_{1} f^{3} / 3+\ldots\right],
$$

where

$$
\begin{aligned}
& f=\left[\left(V_{0} / V_{H}\right)^{2 / 3}-1\right] / 2, \\
& a_{1}=3\left(K_{0 S}^{\prime}-4\right) / 2,
\end{aligned}
$$

and $K_{0 S}$ and $K_{0 S}^{\prime}$ are the bulk modulus and the pressure derivative of the bulk modulus along the principal isentrope, 
respectively. The theoretical values for these two parameters in terms of the shock parameters, $C_{0}$ and $s$, are

$$
\begin{aligned}
& K_{0 S}=C_{0}^{2} \rho_{0}, \\
& \text { and } K_{0 S}^{\prime}=4 s-1 .
\end{aligned}
$$

Once the shock pressure is calculated using Eq. (5), the shock temperature can be calculated in two steps. First, we calculate the temperature along the principal isentrope for the nonporous, crystalline material using the result from Walsh and Christian: ${ }^{34}$

$$
T_{S}=T_{0} \exp \left(-\int_{V_{0}}^{V_{H}} \gamma / V d V\right),
$$

and the shock temperature can then be calculated using

$$
T_{H}=T_{S}+\Delta E / C_{V},
$$

where, $T_{S}$ is the temperature along the principal isentrope defined in Eq. (7) and $C_{V}$ is the heat capacity at constant volume, which can be obtained from Debye model as follows (from Kittel: $:^{35}$ )

$$
C_{V}=9 R\left(\frac{T}{\theta}\right)^{3} \int_{0}^{\theta / T}\left(\frac{x^{4} e^{x}}{\left(e^{x}-1\right)^{2}}\right) d x
$$

where $R$ is the gas constant. The Debye temperature is determined from the measured sound velocity: ${ }^{36}$

$$
\theta=251.2 \sqrt[3]{\frac{3 \rho}{\mu}\left(\frac{2}{v_{s}^{3}}+\frac{1}{v_{p}^{3}}\right)^{-1}},
$$

where $\mu$ is the mean atomic weight. The volume dependence of the Debye temperature can be obtained as following: ${ }^{36}$

$$
\ln \theta=\ln \Theta_{0}+\frac{\Gamma_{0}}{q}\left[1-\left(\frac{V_{H}}{V_{0}}\right)^{q}\right] .
$$

Most materials included in this study have room pressuretemperature elastic moduli available in $C_{i j}$ forms. ${ }^{37} \mathrm{~A}$ Voigt approximation for the bulk modulus and shear modulus was employed to calculate the compressional and shear velocities. Equation (11) assumes the Debye temperature is pressure dependent, but not temperature dependent and the behaviors of the elastic moduli at a high pressure-temperature condition are implicit in this formulation. For those materials which elastic moduli are available to calculate bulk compressional velocity $\left(v_{p}\right)$ and shear velocity $\left(v_{s}\right)$, the Debye model is used. For those materials lacking the sound velocity data, the Dulong-Petit value is assumed.

$\Delta E$ in Eq. (8) is the difference in internal energy generated by the impact $\left(E_{H}=0.5 P_{H} \cdot\left(V_{00}-V_{H}\right)\right.$, from RankineHugoniot relations) and that consumed during compression of the sample from $V_{0}$ to $V_{H}$ along the principal isentrope $\left(\varepsilon_{S}\right)$ as well as that consumed during the phase transition $\left(E_{\mathrm{Tr}}\right.$, if there exists a phase transition), i.e,

$$
\Delta E=E_{H}-\varepsilon_{S}-E_{\mathrm{Tr}} .
$$

Finally, the shock temperature for the porous material can be obtained from modification of the shock temperature of nonporous, crystalline material:

$$
T_{m}=\left(P_{m}-P_{H}\right) \frac{V_{H}}{C_{V} \cdot \gamma}+T_{H},
$$

where $T_{H}, P_{m}$, and $P_{H}$ are defined by Eqs. (8), (5), and (3), respectively. The heat capacities are defined by the Debye model, Eqs. (9) - (11) or by the Dulong-Petit model and the Grüneisen parameter is obtained from Eqs. (4) - (4b).

\section{B. Special considerations on porous materials}

The Hugoniot curves for crystalline materials without shock-induced phase transitions have a concave-upward shape (i.e., $\partial^{2} P / \partial V^{2}>0$ ) in the pressure-volume plane. As the porosity of the materials increases (bulk density decreases), the heat generated due to increasing internal energy makes the material less compressible. At low porosity (i.e., $m>1$ but close to unity), the peak density reached can be greater than that of the crystalline nonporous material. If the porosity has increased, the heat generated via dynamic compression is so great that the material can only be compressed to a density less than the uncompressed nonporous crystalline density. This behavior is often referred as an inverted Hugoniot in many studies and has been observed in many experiments. ${ }^{38,39}$ In Zeldovich and Raiser's ${ }^{4}$ treatment of these Hugoniots, they assumed the Grüneisen parameter to be constant and demonstrated that Eq. (5) described the inverted Hugoniot curves in high porosity material. Trunin et $a l .^{40}$ have treated shock experimental data in porous metals using an equation of state which included electronic contributions, which is necessary for very high-pressuretemperature regimes. In the scope of this study, we allowed the Grüneisen parameter to be volume dependent under all conditions, but the electronic contributions are ignored. In fact, the volume dependence of the Grüneisen parameter $[\gamma$ in Eq. (4)], that of the isentrope temperature [ $T_{S}$ in Eq. (7)], and that of the Debye temperature $[\theta$ in Eq. (11)] were maintained throughout our calculations, even at inverted Hugoniot conditions. This is sufficient for our purpose because the low vaporization threshold observed in our simulations, particularly in the case of minerals.

\section{Entropy calculation}

After calculating the Hugoniot pressure and shock temperature, one can then calculate the entropy associated with each shock state. This method has been used in Yang, ${ }^{41}$ Yang and Ahrens, ${ }^{12}$ and Gupta et al. ${ }^{13}$ to calculate entropies of calcite and anhydrite. Figure 1 shows the Hugoniot curve and the principal isentrope for a model mineral undergoing a single shock-induced phase transformation. In Fig. 1, the Hugoniot curve of this model material consisted a lowpressure phase, a high-pressure phase, and a mixed phase region. We treated the entropy production during the shock compaction as a bulk property (using the heat capacity of the bulk material to calculate the entropy production) and ignored the localized effect due to the presence of hot spots and heterogenous heating. Such an approach is the most conservative estimate of the entropy production, because the existence of these localized hot zones can only increase the total entropy production. Referring to the volume-pressure 


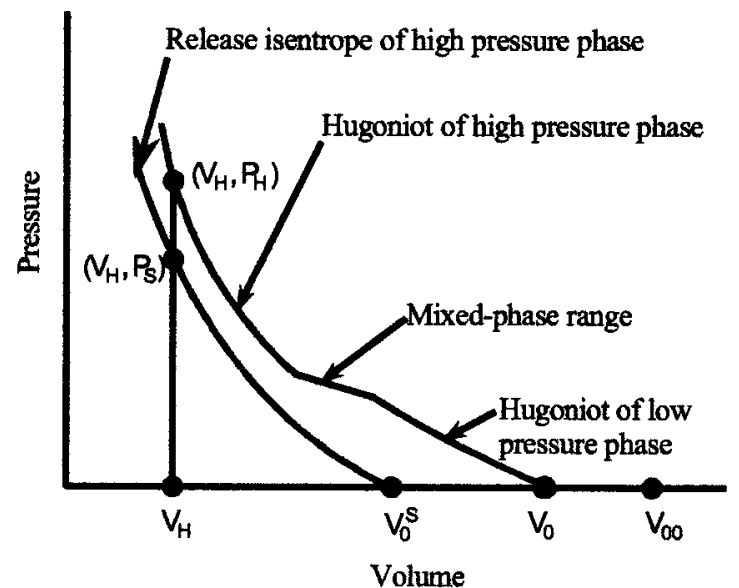

FIG. 1. $P-V$ paths in model material with a single shock-induced phase transition.

points in Fig. 1, the entropy associated with the state $\left(V_{H}, P_{H}\right)$ can be obtained following three $P-V$ paths: (1) from $\left(V_{0}, 1\right.$ bar) to $\left(V_{0}^{S}, 1\right.$ bar), the entropy difference is that due to the phase transition, $\Delta S_{1}=\Delta S_{t r} ;(2)$ from $\left(V_{0}^{S}, 1\right.$ bar $)$ to $\left(V_{H}, P_{S}\right)$, the entropy difference is zero, because the path is along the high-pressure phase principal isentrope, $\Delta S_{2}$ $=0$; and (3) from $\left(V_{H}, P_{S}\right)$ to $\left(V_{H}, P_{H}\right)$, this is isochoric heating and the entropy change is given as $\Delta S_{3}$ $=\int_{T_{S}}^{T_{H}} C_{V} / T d T$, here again the $C_{V}$ is defined by Eqs. (9) to (11) or by the Dulong-Petit value.

Therefore, the entropy associated with the final state ( $\left.V_{H}, P_{H}\right)$ is the sum of $\Delta S_{1}, \Delta S_{2}$, and $\Delta S_{3}$ plus the standard state entropy of the unshocked sample:

$$
S=S^{0}+\Delta S_{1}+\Delta S_{2}+\Delta S_{3} .
$$

\section{Impedance match calculations}

After obtained the Hugoniot pressure from compression, we can calculate the shock velocity and the particle velocity achieved from the Rankine-Hugoniot relations. The shock velocity, $U_{S}$, and the particle velocity, $U_{p}$, can be calculated from:

$$
\begin{aligned}
& U_{S}=V_{00} \cdot \sqrt{\frac{P_{m}}{V_{00}-V_{H}}}, \\
& U_{p}=\sqrt{P_{m} \cdot\left(V_{00}-V_{H}\right)} .
\end{aligned}
$$

The velocity of projectile which induces such given shock state can then be calculated without any knowledge of the shock wave equation of state of the porous materials, which is usually very poorly constrained. Following Ahrens, ${ }^{42}$ the impedance match equation is:

$$
\rho_{0 A}\left(u_{f p}-U_{p}\right)\left[C_{0 A}+s_{A}\left(u_{f p}-U_{p}\right)\right]=\rho_{00} U_{p} U_{S},
$$

where $\rho_{0 A}$ is the density of the projectile, $C_{0 A}, s_{A}$ are the shock wave equation of state parameters for the projectile material, and $u_{f p}$ is the velocity of the projectile just before impact. Using the results from Eqs. (15) and (16), we solve Eq. (17) for the projectile velocity:

$$
u_{f p}=\frac{1}{2}\left[-b+\sqrt{b^{2}-4 c}\right],
$$

where

$$
b=\frac{C_{0 A}-2 s_{A} U_{p}}{s_{A}}
$$

and

$$
c=\frac{1}{s_{A}}\left[s_{A} U_{p}^{2}-\left(C_{0 A}+\frac{\rho_{00}}{\rho_{0 A}} U_{S}\right) U_{p}\right] .
$$

\section{THERMOCHEMICAL DATA AND SHOCK WAVE EQUATIONS OF STATE}

We have conducted calculations on several systems including metals $(\mathrm{Al}, \mathrm{Li}$, and $\mathrm{Fe})$, minerals [calcite $\left(\mathrm{CaCO}_{3}\right)$, dolomite $\left[\mathrm{CaMg}\left(\mathrm{CO}_{3}\right)_{2}\right]$, anhydrite $\left(\mathrm{CaSO}_{4}\right)$, gypsum $\left(\mathrm{CaSO}_{4} \cdot 2 \mathrm{H}_{2} \mathrm{O}\right)$, serpentine $\left[\mathrm{Mg}_{3} \mathrm{Si}_{2} \mathrm{O}_{5}(\mathrm{OH})_{4}\right]$ and brucite $\left.\left(\mathrm{Mg}(\mathrm{OH})_{2}\right)\right]$, ionic solids $(\mathrm{NaCl})$, planetary substances (ice and $\mathrm{FeS}$ ) and organic substances (plexiglas, polymethylmethacrylate, Lexan, and polycarbonate).

We used the Hugoniot data of these materials in the form of their shock wave equation of state parameters $\left(C_{0}\right.$ and $\left.s\right)$, the nature, and density of the high-pressure phase(s) and the parameters describing the Grüneisen parameters simultaneously. The purpose is to generate a self-consistent parameter set for each material (Table I).

In Table I, the values for the initial density of the highpressure phase, $\rho_{0 H}$, were obtained either (1) by taking published values for a single solid phase or (2) by fitting the available Hugoniot data with other listed parameters. The $C_{0}, s$, and " $U_{p}$ range" columns tabulate the parameters of the shock equation of state and the particle velocity range in which the $C_{0}-s$ values were obtained. An iterative procedure $^{30}$ was followed to construct the metastable Hugoniots for the metastable high-pressure phases and phase assemblages for those materials with shock-induced phase transitions. The metastable Hugoniots were used to obtain the associated $C_{0}^{*}-s^{*}$ values (the asterisks represent metastable phases) and are listed in Table I under the $C_{0}$ and $s$ columns. The $q_{0}$ and $q^{\prime}$ columns list the parameters describing the volume dependence of Grüneisen parameters obtained by fitting the available shock wave data. If a material has been studied in porous conditions, $q_{0}$ were obtained by fitting the experimental data at each porosity. An averaged value of $q_{0}$ is tabulated and used in the subsequent calculations. If a particular material does not have experimental Hugoniot data for different porosity, the nonporous $q_{0}$ value is used throughout the calculation. Because $\Gamma_{0}$ can be obtained using Eq. (4b) directly from $s$, therefore, the values of $\Gamma_{0}$ are omitted from Table I.

$\Delta S_{\mathrm{Tr}}$ for a phase transition in Table I was obtained either by (1) calculating from the Clausius-Clapeyron relations ( $\Delta S=\Delta V \cdot d P / d T$ ) or (2) taken from literature. The $E_{\mathrm{Tr}}$ for such transition can be derived from thermodynamic equality: $E_{\mathrm{Tr}}=T_{\mathrm{Tr}} \Delta S_{\mathrm{Tr}}-P_{T r}\left(V_{0}-V_{0}^{L}\right)$. The transition pressures and temperatures were obtained from references indicated in Table I. When insufficient information to obtain $\Delta S_{\operatorname{Tr}}$ and $E_{\mathrm{Tr}}$, exists, these were estimated from thermodynamic prop- 
TABLE I. Summary of parameters used in calculations for solid materials.

\begin{tabular}{|c|c|c|c|c|c|c|c|c|c|c|c|}
\hline $\begin{array}{l}\text { Material. } \\
{[\text { High-pressure phase }(\mathrm{s})]}\end{array}$ & $\begin{array}{c}\rho_{0}{ }^{\mathrm{a}} \\
(\mathrm{g} / \mathrm{cc})\end{array}$ & $\begin{array}{l}\rho_{0 H}{ }^{\mathrm{b}} \\
(\mathrm{g} / \mathrm{cc})\end{array}$ & $\begin{array}{c}C_{0} \\
(\mathrm{~km} / \mathrm{s})\end{array}$ & $s$ & $\begin{array}{l}U_{p} \text { range } \\
(\mathrm{km} / \mathrm{s})\end{array}$ & $q_{0}$ & $q^{\prime}$ & $\begin{array}{c}E_{\operatorname{Tr}} \\
(\mathrm{kJ} / \mathrm{mol})\end{array}$ & $\begin{array}{c}\Delta S_{\operatorname{Tr}} \\
\left(\mathrm{J} . \mathrm{mol}^{-1} \mathrm{~K}^{-1}\right)\end{array}$ & $\begin{array}{c}S_{0} \\
\left(\mathrm{~J} \cdot \mathrm{mol}^{-1} \mathrm{~K}^{-1}\right)\end{array}$ & Reference No. \\
\hline $\mathrm{Al}^{\mathrm{c}}$ (none) $)^{\mathrm{d}}$ & 2.788 & 2.788 & 5.356 & 1.305 & $0.0-5.96$ & 1.20 & 0.77 & 0 & 0 & 28.425 & 43,44 \\
\hline $\mathrm{Fe}(\varepsilon-\mathrm{Fe})$ & 7.860 & 8.28 & 3.94 & 1.58 & $1.41-4.55$ & 1.32 & 0.89 & 5 & 1.3 & 27.476 & $43,44,45$ \\
\hline $\mathrm{Li}$ (none) & 0.535 & 0.535 & 4.58 & 1.15 & $0.0-4.81$ & 1.17 & 0.8 & 0 & 0 & 29.237 & 44,46 \\
\hline $\mathrm{NaCl}$ (B2 phase) & 2.159 & 2.232 & 2.43 & 1.66 & $2.5-3.75$ & 1.28 & 0.9 & 35 & 1.8 & 72.115 & $43,44,47,48$ \\
\hline $\mathrm{FeS}(\mathrm{FeS}-\mathrm{IV})$ & 4.605 & 4.94 & 2.947 & 1.578 & $1.59-3.99$ & 1.38 & 0.9 & 23.3 & 48.2 & 60.33 & $44,49,50$ \\
\hline $\begin{array}{l}\text { Anhydrite }\left(\mathrm{CaSO}_{4}\right) \\
\left(\mathrm{CaO}+\mathrm{SO}_{3}\right)\end{array}$ & 2.970 & 2.995 & 3.25 & 1.72 & $2.42-3.71$ & 1.12 & 0.92 & 11.6 & 32.7 & 106.69 & $43,44,51,41$ \\
\hline $\begin{array}{l}\text { Gypsum }(\mathrm{CaSO} 4 \cdot 2 \mathrm{H} 2 \mathrm{O}) \\
\left(\mathrm{CaO}+\mathrm{SO}_{3}+2 \mathrm{H}_{2} \mathrm{O}\right)\end{array}$ & 2.280 & 2.29 & 2.49 & 1.79 & $2.15-4.06$ & 1.07 & 0.9 & (54) & (74) & 194.14 & $43,44,51$ \\
\hline $\begin{array}{l}\text { Calcite }\left(\mathrm{CaCO}_{3}\right) \\
\left(\mathrm{CaO}+\mathrm{CO}_{2}\right)\end{array}$ & 2.701 & 2.75 & 3.71 & 1.435 & $0.81-3.84$ & 1.68 & 1.0 & 20 & 6.7 & 91.71 & $43,44,41,52,53$ \\
\hline $\begin{array}{l}\text { Dolomite }\left(\mathrm{CaMg}\left(\mathrm{CO}_{3}\right)_{2}\right) \\
\left(\mathrm{CaO}+\mathrm{MgO}+2 \mathrm{CO}_{2}\right)\end{array}$ & 2.828 & 2.89 & 5.3 & 1.16 & $1.12-5.32$ & 1.74 & 1.0 & (50) & (14) & 155.18 & $43,44,53$ \\
\hline Ice $\left(\mathrm{H}_{2} \mathrm{O}, 263 \mathrm{~K}\right)$ (liquid water) & 0.950 & 0.999 & 2.64 & 1.27 & $2.47-32.4$ & 0.76 & 0.5 & 32.1 & 43.6 & 37.71 & $43,44,54,55$ \\
\hline $\begin{array}{l}\text { Brucite }\left(\mathrm{Mg}(\mathrm{OH})_{2}\right) \\
\left(\mathrm{MgO}+\mathrm{H}_{2} \mathrm{O}\right)\end{array}$ & 2.391 & 2.391 & 4.76 & 1.35 & $1.25-3.41$ & 1.19 & 0.8 & $\sim 0$ & $\sim 0$ & 63.18 & $43,44,51,56$ \\
\hline $\begin{array}{l}\text { Serpentine }\left(\mathrm{Mg}_{3} \mathrm{Si}_{2} \mathrm{O}_{5}(\mathrm{OH})_{4}\right) \\
\left(\mathrm{Mg}_{2} \mathrm{SiO}_{4}+\mathrm{MgSiO}_{3}+2 \mathrm{H}_{2} \mathrm{O}\right)\end{array}$ & 2.620 & 2.63 & 3.80 & 1.34 & $2.66-5.43$ & 0.97 & 0.62 & $(28.5)$ & $(47)$ & 221.3 & $43,44,57,58$ \\
\hline $\begin{array}{l}\text { Lexan (Polycarbonate) } \\
\text { (unknown) }\end{array}$ & 1.193 & 1.25 & 2.617 & 1.28 & $3.64-6.92$ & 0.88 & 0.55 & $(60)$ & $(50)$ & $(300)$ & 43,46 \\
\hline PMMA (unknown) & 1.180 & 1.23 & 3.167 & 1.3 & $2.6-10.2$ & 0.76 & 0.5 & $(60)$ & $(50)$ & $(300)$ & 46,59 \\
\hline
\end{tabular}

${ }^{2}$ Density of low-pressure phase.

${ }^{\mathrm{b}}$ Bulk density of the high-pressure phase or phase assembly.

${ }^{c}$ Low-pressure phase.

${ }^{\mathrm{d}}$ High-pressure phase or phase assembly

erties of materials having similar chemical formula and crystal structure. These were labeled within a parenthesis. For brucite, the high-pressure phase assemblage $\left(\mathrm{MgO}+\mathrm{H}_{2} \mathrm{O}\right)$ has a molar volume very close to that of the brucite. As a result, no discontinuity is seen in the $U_{s}-U_{p}$ plot for brucite $^{56}$ and thus the $\Delta S_{\mathrm{Tr}}$ and $E_{\mathrm{Tr}}$ terms were nearly zero. The $S_{0}$ column is the standard entropy of the low-pressure phase at $298 \mathrm{~K}$ and the values were taken from the NISTJANAF tables ${ }^{60}$ or from Robie et al. ${ }^{44}$

\section{RESULTS AND DISCUSSIONS}

The calculated Hugoniot curves of selected materials (aluminum, calcite, ice at $263 \mathrm{~K}$, and $\mathrm{NaCl}$ ) are compared with the experimental data and are tabulated in Table II. The values in parentheses are our modeling results. Table II calculated the Hugoniot pressures and $U s-U p$ relations agree with published Hugoniot data within $1 \%$ at distentions $<2$ and to $5 \%$ at distentions $>2$. The calculated entropies for

TABLE II. Comparison of calculated Hugoniot and published Hugoniot data.

\begin{tabular}{lccccc}
\hline \hline Material & $m$ & $\eta$ & $P_{m}(\mathrm{GPa})$ & $U_{s}(\mathrm{~m} / \mathrm{s})$ & $U_{p}(\mathrm{~m} / \mathrm{s})$ \\
\hline $\mathrm{Al}^{\mathrm{a}, \mathrm{b}}$ & $1.430(1.420)^{\mathrm{a}}$ & $0.332(0.333)$ & $139.1(143.0)$ & $11740(11738)$ & N/A $(6222)$ \\
& $2.080(2.080)$ & $0.150(0.146)$ & $100.3(95.1)$ & $11420(10966)$ & N/A (6466) \\
& $2.980(2.980)$ & $0.0148(0.0151)$ & $70.2(63.6)$ & $10750(10073)$ & N/A (6744) \\
Calcite $^{\mathrm{c}}$ & $1.319(1.318)$ & $0.313(0.314)$ & $71.1(66.8)$ & $8420(8167)$ & $4180(3978)$ \\
& $1.320(1.318)$ & $0.176(0.178)$ & $19.0(19.1)$ & $4870(4911)$ & $1930(1895)$ \\
& $1.563(1.518)$ & $0.256(0.258)$ & $59.4(62.9)$ & $8030(8261)$ & $4340(4423)$ \\
$\mathrm{Ice}^{\mathrm{d}}$ & $1.093(1.1095)$ & $0.504(0.500)$ & $37.7(36.3)$ & $8520(8595)$ & $4840(4863)$ \\
& $1.667(1.665)$ & $0.389(0.389)$ & $35.4(39.8)$ & $9520(10357)$ & $6200(6744)$ \\
& $2.857(2.864)$ & $0.155(0.157)$ & $22.2(22.9)$ & $9390(9799)$ & $6750(7054)$ \\
$\mathrm{NaCl}^{\mathrm{e}}$ & $1.00(1.00)$ & $0.385(0.387)$ & $48.5(48.8)$ & $7446(7451)$ & $3009(3031)$ \\
& $1.514(1.522)$ & $0.158(0.157)$ & $16.2(15.8)$ & $4950(4896)$ & $2290(2272)$ \\
& $2.185(2.180)$ & $0.025(0.025)$ & $11.2(9.9)$ & $4450(4097)$ & $2530(2343)$ \\
\hline \hline
\end{tabular}

${ }^{\mathrm{a}}$ Numbers in parentheses are calculated in this study.

${ }^{\mathrm{b}}$ See Ref. 38.

${ }^{\mathrm{c}}$ See Ref. 53.

${ }^{\mathrm{d}}$ See Ref. 54.

${ }^{\mathrm{e} S e e}$ Refs. 46 and 47.

${ }^{\mathrm{f}} \mathrm{N} / \mathrm{A}$ indicates not applicable 


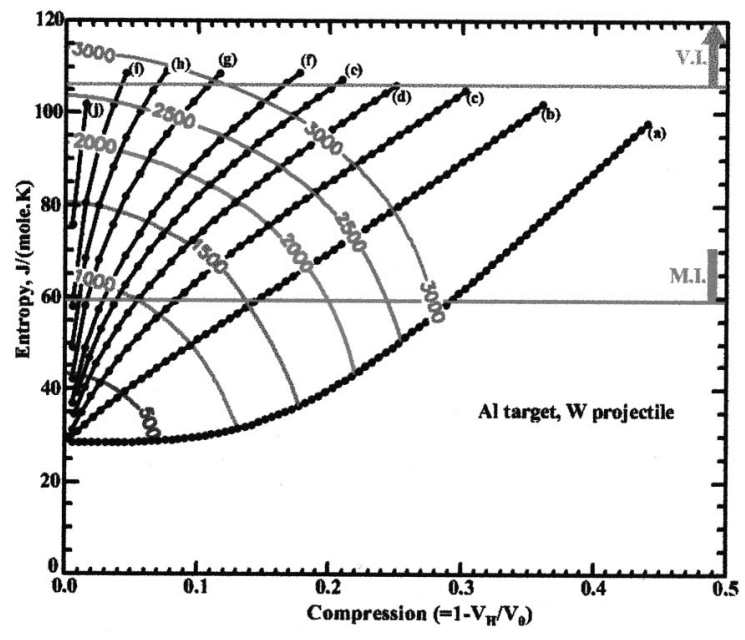

FIG. 2. Calculated entropy of shocked aluminum using tungsten projectile. Contours show constant projectile velocity in every $500 \mathrm{~m} / \mathrm{s}$. The distention associated with labeled Hugoniot is (a) 1.0, (b) 1.1, (c) 1.2, (d) 1.3, (e) 1.4, (f) 1.5, (g) 1.7 , (h) 1.9 , (i) 2.2, and (j) 2.5.

these four substances (aluminum, $\mathrm{CaCO}_{3}$, ice, and $\mathrm{NaCl}$ ) impacted by tungsten projectile are plotted in Figs. 2-5. Similar colored plots for all substances and other projectile materials (304 stainless steel and aluminum) are available from the Electronic Physics Auxiliary Publication Service (EPAPS) of the American Institute of Physics (AIP). ${ }^{61}$ Figures $2-5$ plotted entropy versus compression with distentions as a parameter. Each plot is also superimposed with the contours of the projectile velocity. The contours were obtained using an interpolation routine. The contours in Figs. 2-5 have a convex-upward shape and become flattened as the distention increases, which may be explained by the two competing processes theory proposed by Ahrens. ${ }^{24}$ These competing processes were: (1) an increase in the production of entropy (heat production) and (2) a decrease in the impedance matching as the porosity increases. According to Ahrens $^{24}$ and Ahrens and O'Keefe, ${ }^{25}$ an optimal entropy-

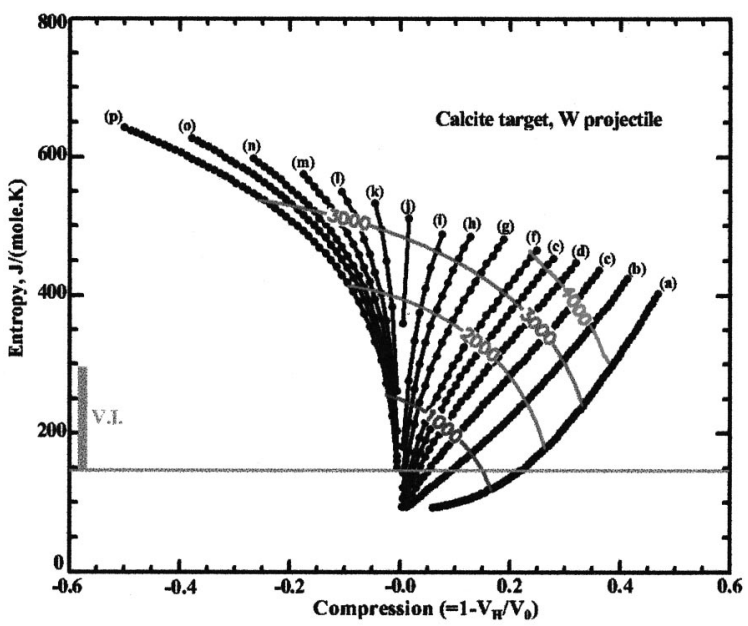

FIG. 3. Calculated entropy of shocked calcite $\left(\mathrm{CaCO}_{3}\right)$ using tungsten projectile. Contours show constant projectile velocity in every $1000 \mathrm{~m} / \mathrm{s}$. The distention associated with labeled Hugoniot is (a) 1.0, (b) 1.1, (c) 1.2, (d) 1.3 , (e) 1.4 , (f) 1.5 , (g) 1.7 , (h) 1.9, (i) 2.2, (j) 2.8, (k) 3.1, (1) 3.5, (m) 3.8, (n) 4.3 , and (o) 4.9 .

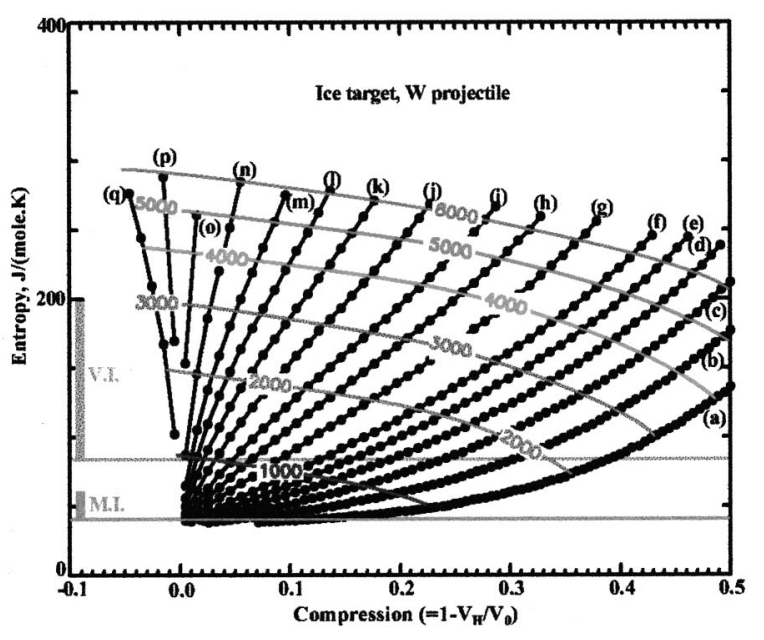

FIG. 4. Calculated entropy of shocked ice (initial temperature at $263 \mathrm{~K}$ ) using tungsten projectile. Contours show constant projectile velocity in every $1000 \mathrm{~m} / \mathrm{s}$. The distention associated with labeled Hugoniot is (a) 1.0, (b) 1.1 , (c) 1.2 , (d) 1.3 , (e) 1.4 , (f) 1.5 , (g) 1.7 , (h) 1.9 , (i) 2.1 , (j) 2.4, (k) 2.7, (l) $3.0,(\mathrm{~m}) 3.4$, (n) 3.9, and (o) 4.7 .

generating distention for a certain impact velocity was observed. We did not observe such an optimal distention in any of the materials studied in our calculations even though our calculations covered a much larger distention and compression range, but the flattening of the projectile velocity contours does suggest that the competing processes theory is still valid within the compression and distention range covered by this study. We attributed this discrepancy in observation of the optimal entropy-generating distention to the different approaches used in the impedance match calculations. However, since we limited the distention range in our calculation from 1 to 5 and the possibility of an optimal distention occurring at even higher distentions cannot be completely ruled out. Such an investigation will require a better Grüneisen parameter model, because of the amount of heat produced (shock temperatures well over $10000 \mathrm{~K}$ ). In addition, the

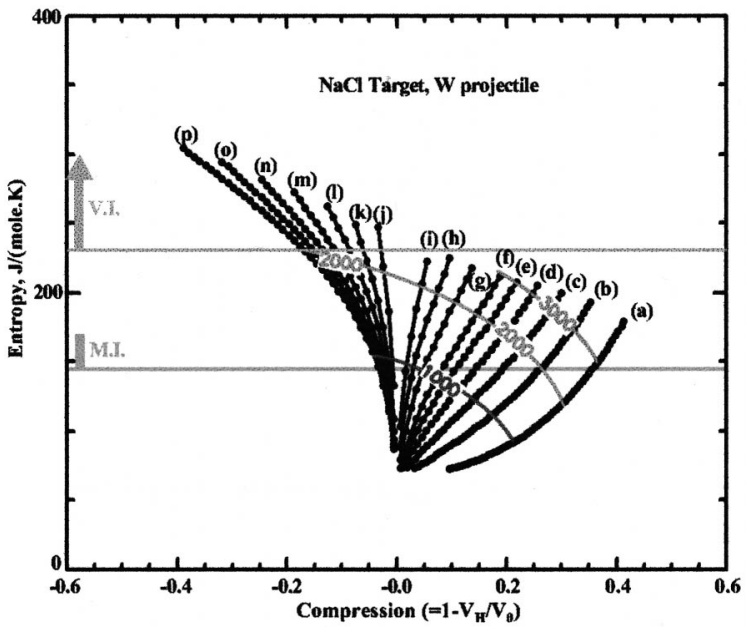

FIG. 5. Calculated entropy of shocked $\mathrm{NaCl}$ using tungsten projectile. Contours show constant projectile velocity in every $1000 \mathrm{~m} / \mathrm{s}$. The distention associated with labeled Hugoniot is (a) 1.0, (b) 1.1, (c) 1.2, (d) 1.3, (e) 1.4, (f) 1.5, (g) 1.7, (h) 1.9, (i) 2.3 , (j) 2.6, (k) 3.0, (1) 3.4, (m) 3.8, (n) 4.3, and (o) 4.8 . 
existence of heterogeneous heating and hot spots may enhance the effect of one aspect of the competing process. The high temperatures at the surface of the grains generated by these nonlinear processes ${ }^{28}$ should greatly decrease the strength of the porous material and will probably enhance the impedance contrast to increase more dramatically. The enhancement on the entropy production may be limited due to the finite amount of internal energy supplied.

In Figs. 2-5 (including those in the Deposit), the vaporization intervals and melting intervals were labeled as V.I. and M.I. and additional horizontal lines were drawn to facilitate visualizing the beginning of vaporization and melting. These intervals were obtained from various sources and methods. For metals, these values were calculated using the NIST-JANAF tables. ${ }^{60}$ For minerals, the species present in the vapor were assumed and an estimated vaporization temperature were calculated. ${ }^{13}$ We then calculated the entropy of the solid and vapor at the vaporization temperature. Some materials go directly into vapor phase or decompose, thus only the vaporization interval is shown. For aluminum with distention $<1.9$, a minimum projectile velocity of $2.7 \mathrm{~km} / \mathrm{s}$ is needed for initiating vaporization using a tungsten projectile. Similar projectile speeds are needed to vaporize iron and lithium. For ionic solids such as $\mathrm{NaCl}$, the required impact velocity for initiating vaporization is somewhat lower than that for metals. In the case of $\mathrm{NaCl}$ at distention $<2.2,2.5$ $\mathrm{km} / \mathrm{s}$ is needed to drive the system into the vapor state. For volatile-bearing minerals, the minimum impact velocities are much lower, ranging from less than 1 to $1.5 \mathrm{~km} / \mathrm{s}$. The implication of low projectile velocity is that researchers can reasonably expect that with a properly designed mass spectrometer, an impact experiment using gun launched projectile will provide sufficient energy for vaporizing mineral specimens upon release from the shocked state.

Some approximations and assumptions were made during the modeling and these may contribute uncertainty and errors to the calculation results. A major source of error is the assumption of $C_{v}$ being Debye-type or Dulong-Petit value. The applicability of Dulong-Petit rule in minerals and polymers are very suspicious, especially in the low temperature region $\left(T<\Theta_{D}\right)$. In fact, many mineral physical literatures showed that even the Debye model for the heat capacity was not entirely satisfactory in common minerals and a modified lattice vibration model was introduced. ${ }^{62}$ In the past two decades, we have seen better thermodynamic databases of minerals become available, ${ }^{63}$ but thermodynamic properties of many high-pressure phases are nonexisting. Furthermore, for polymers, knowledge of the nature of the high-pressure phases are extremely limited and not to mention the thermodynamic properties. In fact, for those materials with elastic properties, the discrepancy in constant volume heat capacities calculated using the Debye model and the Dulong-Petit model at any distention within the calculation grid and at high temperatures (greater than the Debye temperatures) is always less than $1 \%$. Take calcite for example, the calculated constant pressure heat capacity $(C p)$ at $3000 \mathrm{~K}$, using the Debye model yielded $165.4 \mathrm{~J} / \mathrm{K} \mathrm{mol}$, the value calculated using the Dulong-Petit model yielded $165.6 \mathrm{~J} / \mathrm{K} \mathrm{mol}$, and the thermochemical database ${ }^{63}$ showed a value of 159.6
$\mathrm{J} / \mathrm{K}$.mol, an error of $3.7 \%$. The error at the low-temperature end is much higher. Using calcite as an example again, the constant pressure heat capacity in the thermochemical database $^{63}$ at $300 \mathrm{~K}$ is $84.9 \mathrm{~J} / \mathrm{K}$ mol, the Dulong-Petit model yielded $125.3 \mathrm{~J} / \mathrm{K}$ mol and the Debye model yielded 107.7 $\mathrm{J} / \mathrm{K}$ mol. The Debye model is about $26.8 \%$ off and the Dulong-Petit model is about $47.5 \%$ off. Calcite is one of the extreme example due to the non-Debye behavior. ${ }^{36}$ Even though the discrepancies seem huge, but the temperature range is limited where these unbearable discrepancy exists. Therefore, the entropy calculation should incur small differences and comparing the difference between the entropies calculated using the Dulong-Petit model and Debye model did yield very similar values (mostly $<1 \%$ ). Additionally, in most of our calculation the temperature range well exceeds the Debye temperature, then the discrepancy is much better controlled. We argue that these heat capacities calculated using either the Dulong-Petit model or Debye model are still quite good first-order approximation for the high-pressure phases.

The other assumption, which may have considerable effect on the results, is the Grüneisen parameter model. In limited pressure-temperature range, the assumption of a simple volume dependency is acceptable. However, as the shock temperature increases, the assumption ceases to be a good description of the solid due to the increase electronic contribution to the heat capacity. At high temperatures, the electrons started to take up a significant amount of thermal energy and an electron correction term is generally needed for the Grüneisen parameter. From Eliezer et al. ${ }^{26}$ the temperature for the electrons to become classical is about $10^{4} \mathrm{~K}$, therefore, we limited our calculations to be within $10^{4} \mathrm{~K}$ and thus our assumption of volume dependency of the Grüneisen parameter should hold.

The stability issue must be addressed by free energy calculations. We followed the approach by Gupta et al. ${ }^{13}$ to calculate the entropy associated with incipient and complete vaporization by extrapolating available thermodynamic data to high temperatures to estimate the vaporization temperature and then evaluate the entropy of the solid and the vapor phases at the vaporization temperature. In this study, we simply assumed the impact ionization is far less common than impact vaporization, because the energy required for ionizing the vapor species are far too great. The first ionization energy in Cs is a few electron volts and other atoms have an even higher first ionization energy. Therefore, even though it is possible to have some ionized species in the ejecta, the amount of the ionized species can be very small. In addition, the lifetime of these ionized species can be rather short. Therefore, assuming neutral species should be a very good first approximation. The major problem, in fact, is that actual vaporization species are unknown. Some recent literature do suggest that the ejecta has a very different, even nonstoichiometric, chemical composition from the bulk material. ${ }^{64}$ However, the equilibrium approach is a much simpler and the most conservative estimate of the vapor generated, as indicated by many studies in hot spot and heterogeneous heating have been able to estimate the anomalous temperature increase and the promoted kinetics. Our current understanding 
of the speciation reactions in the shock-induced vapors are extremely poor and further experimental study of the speciations in the vapors will be tremendously valuable in elucidating the shock-induced vaporization processes.

\section{ACKNOWLEDGMENTS}

Research supported by NASA and DOE. This is contribution No. 8876, Division of Geological and Planetary Sciences, California Institute of Technology, Pasadena, CA 91125.

${ }^{1}$ W. Alvarez, E. G. Kauffman, F. Surlyk, L. W. Alvarez, F. Asaro, and H. V. Michel, Science 223, 1135 (1984).

${ }^{2}$ R. Brett, Geochim. Cosmochim. Acta, 56, 3603 (1992)

${ }^{3}$ B. Fegley, R. G. Prinn, H. Martman, and G. H. Watkins, Nature (London) 319, 305 (1986).

${ }^{4}$ Y. B. Zeldovich and Y. P. Raiser, Physics of Shock Waves and Hightemperature Hydrodynamic Phenomena (Academic, New York, 1967), p. 880.

${ }^{5}$ E. Pierazzo and H. J. Melosh, Annu. Rev. Earth Planet Sci. 28, 141 (2000).

${ }^{6}$ K. O. Pope, K. H. Baines, A. C. Ocampe, and B. A. Ivanov, J. Geophys. Res. 102, 21645 (1997).

${ }^{7}$ M. B. Boslough, T. J. Ahrens, J. Vizgirda, R. H. Becker, and S. Epstein, Earth Planet. Sci. Lett. 61, 166 (1982).

${ }^{8}$ J. A. Tyburczy, R. V. Krishnamurthy, S. Epstein, and T. J. Ahrens, Earth Planet. Sci. Lett. 98, 245 (1990); J. A. Tyburczy, X. Xu, T. J. Ahrens, and S. Epstein, Earth Planet. Sci. Lett. 192, 23 (2001).

${ }^{9}$ J. Kissel and F. R. Krueger, Appl. Phys. A: Solids Surf. 42, 69 (1987)

${ }^{10}$ K. Hornung and J. Kissel, Astron. Astrophys. 291, 324 (1994).

${ }^{11}$ D. E. Austin, T. J. Ahrens, and J. L. Beachamp, Rev. Sci. Instrum. 73, 185 (2001).

${ }^{12}$ W. Yang and T. J. Ahrens, Earth Planet. Sci. Lett. 156, 125 (1998).

${ }^{13}$ S. C. Gupta, T. J. Ahrens, and W. Yang, Earth Planet. Sci. Lett. 188, 399 (2001).

${ }^{14}$ J. D. O'Keefe and T. J. Ahrens, Geochim. Cosmochim. Acta 8, 3357 (1977).

${ }^{15}$ E. Pierazzo and H. J. Melosh, Earth Planet. Sci. Lett. 165, 163 (1999).

${ }^{16}$ F. J. Krieger, Rand Co. Memorandum RM-4590, (Rand Co.Santa Monica, CA, 1965), p. 29.

${ }^{17}$ F. J. Krieger, Rand Co. Memorandum RM-5248, (Rand Co.Santa Monica, CA, 1967), p. 29.

${ }^{18}$ F. J. Krieger, Rand Co. Memorandum RM-5337, (Rand Co.Santa Monica, CA, 1967), p. 45.

${ }^{19}$ F. J. Krieger, Rand Co. Memorandum RM-5592, (Rand Co.Santa Monica, CA, 1968), p. 30.

${ }^{20}$ F. J. Krieger, Rand Co. Memorandum RM-5876, (Rand Co.Santa Monica, CA, 1968), p. 34.

${ }^{21}$ F. J. Krieger, Rand Co. Memorandum RM-6335, (Rand Co.Santa Monica, CA, 1970), p. 31.

${ }^{22}$ L. V. Zhigilei and B. J. Garrison, J. Appl. Phys. 88, 1281 (2000).

${ }^{23}$ L. V. Zhigilei, P. B. S. Kodali, and B. J. Garrison, J. Phys. Chem. B 102, 2845 (1998).

${ }^{24}$ T. J. Ahrens, J. Appl. Phys. 43, 2443 (1972).

${ }^{25}$ T. J. Ahrens and J. D. O'Keefe, Moon 4, 214 (1972).

${ }^{26} \mathrm{~S}$. Eliezer, A. Ghatak, H. Hora, and E. Teller, An Introduction to Equations of State - Theory and Applications (Cambridge University Press, Cambridge, UK 1986), p. 366.

${ }^{27}$ D. D. Dlott and M. D. Fayer, J. Chem. Phys. 92, 3798 (1990); A. Tokmakoff, M. D. Fayer, and D. D. Dlott, J. Phys. Chem. 97, 1901 (1993).

${ }^{28}$ T. C Germann, B. L. Holian, P. S. Lomdahl, A. J. Heim, N. GronbechJensen, and J. B. Maillet, Extended abstract, Proceedings of the 12th International Detonation Symposium, San Diego, CA (2002).

${ }^{29}$ V. F. Nesterenko, Dynamics of Heterogeneous Materials (Springer, Berlin, 2001), p. 510; Chap. 5.

${ }^{30}$ R. G. McQueen, J. N. Fritz, and S. P. Marsh, J. Geophys. Res. 68, 2319 (1963).
${ }^{31}$ R. Jeanloz, J. Geophys. Res. 94, 5873 (1989).

${ }^{32}$ T. J. Ahrens, in High Pressure Shock Compression of Solids, edited by J. R. Asay and M. Shahinpoor (Springer, Berlin, 1993), pp. 75-114.

${ }^{33}$ A. L. Ruoff, J. Appl. Phys. 38, 4976 (1967).

${ }^{34}$ J. M. Walsh and R. H. Christian, Phys. Rev. 97, 1544 (1955).

${ }^{35}$ C. Kittel, Introduction to Solid State Physics, 6th ed. (Wiley, New York, 1986), p. 646.

${ }^{36} \mathrm{O}$. L. Anderson, Equations of State of Solids for Geophysics and Ceramic Sciences (Oxford University Press, New York, 1995), p. 405.

${ }^{37}$ R. Bechmann, R. F. S. Hearmon, S. K. Kurtz, K. H. Herausgeber, and A. M. Hellwege, Elastic, Piezoelectric, Piezooptic, Electrooptic Constants, and Nonlinear Dielectric Susceptibilities of Crystals (Springer, Berlin, 1969), p. 232.

${ }^{38}$ S. B. Kormer, A. I. Funtikov, V. D. Urlin, and A. N. Kolesnikova, Zh. Eksp. Teor. Fiz. 42, 686 (1962) [Sov. Phys. JETP 15, 477 (1962)].

${ }^{39}$ K. K. Krupnikov, M. I. Brazhnik, and V. P. Krupnikova, Zh. Eksp. Teor. Fiz. 42, 675 (1962) [Sov. Phys. JETP 15, 470 (1962)].

${ }^{40}$ R. F. Trunin, A. B. Medvedev, A. I. Funtikov, M. A. Podurets, G. V. Simakov, and A. G. Sevastyanov, Zh. Eksp. Teor. Fiz. 95, 631 (1989) [Sov. Phys. JETP 68, 356 (1989)].

${ }^{41}$ W. Yang, Ph.D. thesis, California Institute of Technology, 1996.

${ }^{42}$ T. J. Ahrens, Methods of Experimental Physics (Academic, New York, 1987), Vol. 24A, pp. 185-235.

${ }^{43}$ T. J. Ahrens and M. J. Johnson, in Mineral Physics and Crystallography: A Handbook of Physical Constants, edited by T. J. Ahrens (American Geophysical Union, Washington, DC, 1995), pp. 143-184.

${ }^{44}$ R. A. Robie, B. S. Hemingway, and J. R. Fisher, (U. S. Geological Survey Bulletin (1978), Vol. 1452, p. 456.

${ }^{45}$ A. I. Funtikov, Fiz. Zemli. 11, 70 (2000) [Izv. Phys. Solid Earth 36, 958 (2000)].

${ }^{46}$ LASL Shock Hugoniot Data edited by S. P. Marsh (University of California Press, Berkeley, 1980), p. 658.

${ }^{47}$ S. B. Kormer, V. Sinitsyn, A. I. Funtikov, V. D. Urlin, and A. V. Blinov, Zh. Eksp. Teor. Fiz. 47, 1202 (1965) [Sov. Phys. JETP 20, 811 (1965)].

${ }^{48}$ D. R. Schmitt, T. J. Ahrens, and B. Svendsen, J. Appl. Phys. 63, 99 (1988).

${ }^{49}$ J. M. Brown, T. J. Ahrens, and D. L. Shampine, J. Geophys. Res. 89, 6041 (1984).

${ }^{50}$ Y. Fei, C. T. Prewitt, H. K. Mao, and C. M. Bertka, Science 268, 1892 (1995).

${ }^{51}$ G. V. Simakov, M. N. Pavlovskiy, N. G. Kalashnikov, and R. F. Trunin, Izv. Earth Phys. (USSR) 8, 11 (1973) [Izv. Earth Phys. 10, 488 (1974)].

${ }^{52}$ B. A. Ivanov and A. Deutsch, Abstract No. 1740, Proceedings of the 32nd Lunar and Planetary Science Conference, Houston, TX, 2001.

${ }^{53}$ N. G. Kalashnikov, M. N. Pavlovskiy, G. V. Simakov, and R. F. Trunin, Izv. Earth Phys. (USSR) 2, 23 (1973) [Izv. Earth Phys. 4, 80 (1973)].

${ }^{54}$ A. A. Bakanova, V. N. Zubarev, Y. N. Sutulov, and R. F. Trunin, Zh. Eksp. Teor. Fiz. 68, 1099 (1975) [Sov. Phys. JETP 41, 544 (1976)].

${ }^{55}$ Stewart, S. T., Ph.D. thesis, California Institute of Technology, Pasadena, CA, 2002.

${ }^{56}$ T. S. Duffy, T. J. Ahrens, and M. A. Lange, J. Geophys. Res. 96, 14319 (1991).

${ }^{57}$ J. A. Tyburczy, T. S. Duffy, T. J. Ahrens, and M. A. Lange, J. Geophys. Res. 96, 18011 (1991).

${ }^{58}$ E. A. Kozlov et al., Chem. Phys. Rep. 14, 208 (1995).

${ }^{59} \mathrm{M}$. Van Thiel, Compendium of Shock Wave Data (University of California, Lawrence Livermore Laboratory, UCRL-50801) (1977).

${ }^{60}$ NIST-JANAF Thermochemical Tables, J. Phys. Chem. Ref. Data, Monograph 9 (American Chemical Society and American Institute of Physics, Washington, DC, 1998), p. 1951.

${ }^{61} \mathrm{ftp} / / / \mathrm{ftp}$. aip.org/epaps/journ_appl_phys/E-JAPIAU-93-058309/

${ }^{62}$ S. W. Kieffer, Rev. Geophys. Space Phys. 17, 1 (1979).

${ }^{63}$ Saxena et al., Thermodynamic Data on Oxides and Silicates: An Assessed Data Set Based on Thermochemistry and High Pressure Phase Equilibrium, (Springer, Berlin, 1993), p. 428

${ }^{64}$ M. V. Gerasimov, B. A. Ivanov, O. I. Yakovlev, and Y. P. Dikov, Earth, Moon, Planets 80, 209 (1998). 